\title{
Combined Infliximab and Rituximab in Necrotising Scleritis
}

\author{
Jiten Morarji ${ }^{a, b} \quad$ Lavnish Joshi $^{a-c}$ Oren Tomkins-Netzer ${ }^{a, b}$ \\ Sue Lightman ${ }^{a, c}$ Simon R.J. Taylor ${ }^{a, b, d}$ \\ ${ }^{a}$ Multidisciplinary Vasculitis Clinic, Imperial College Healthcare NHS Trust, \\ Hammersmith Hospital, London, ${ }^{b}$ Royal Surrey County Hospital NHS Foundation \\ Trust, Guildford, ' UCL Institute of Ophthalmology, and ${ }^{\mathrm{d}}$ Division of Immunology \\ and Inflammation, Faculty of Medicine, Imperial College London, London, UK
}

\section{Key Words}

Infliximab · Rituximab - Granulomatosis with polyangiitis · Scleritis

\begin{abstract}
We report a patient with necrotising scleritis in whom infliximab was used for short-term steroid-sparing while rituximab took effect. This enabled disease control without requiring an extended period of high-dose corticosteroid administration or the concurrent use of cyclophosphamide.
\end{abstract}

\section{Introduction}

Granulomatosis with polyangiitis (GPA), previously known as Wegener's granulomatosis, is a multi-system autoimmune disease, often associated with antineutrophil cytoplasm antibodies (ANCA), which commonly involves the eye as well as the respiratory tract and kidneys. Ophthalmic complications occur in $50-60 \%$ of patients and typically take the form of necrotising scleritis or granulomatous orbital involvement [1]. Ophthalmologists need to be aware that these conditions are potentially sight-threatening - some $90 \%$ of patients with necrotising scleritis develop ocular complications and 50\% develop significant visual loss [2]. Suboptimal treatment of either condition can lead to serious visual consequences.

Cyclophosphamide and corticosteroids have been the cornerstones of treatment for severe GPA but are associated with treatment-related morbidity and mortality [3]. More recent treatment options include the biological agent rituximab, which is a monoclonal antibody directed against CD20, a cell surface antigen expressed by many

Simon R.J. Taylor, MA, PhD, FRCOphth
Faculty of Medicine, Imperial College London Room 5N8B, 5th Floor Commonwealth Building

Hammersmith Hospital, London W12 0NN (UK) E-Mail s.r.taylor@imperial.ac.uk 
subtypes of B cells, although not by plasma cells. We have recently shown that rituximab can be effective in refractory ophthalmic GPA and induce long-lasting disease remission, but it takes up to 6 months to work, meaning that additional agents may be required to provide disease control in the interim [4].

Infliximab belongs to a different class of biological agents, being a monoclonal antibody directed against tumour necrosis factor-alpha (TNF- $\alpha$ ). It is licensed for the treatment of both rheumatoid arthritis and Crohn's disease in the United States and Europe [5] and has also demonstrated efficacy in the treatment of the ANCA-associated vasculitides (AAV), such as GPA, although a recent study in which infliximab was added to standard therapy in patients with active AAV failed to demonstrate any benefit compared to standard therapy alone [6]. Infliximab has been used successfully in the treatment of refractory scleritis $[7,8]$.

We report here a patient with necrotising scleritis associated with GPA in whom infliximab was used for short-term steroid-sparing while rituximab took effect. This enabled disease control without requiring an extended period of high-dose corticosteroid administration or the concurrent use of cyclophosphamide, neither of which was desirable as a result of his comorbidities and previous treatment history.

\section{Case Report}

A 71-year-old man was referred to the Hammersmith Hospital Multidisciplinary Vasculitis Clinic with necrotising scleritis in the left eye ( $\underline{\text { fig. } 1}$ ). He had previously been diagnosed with GPA on the basis of characteristic renal disease associated with a raised ANCA titre and the presence of anti-PR3 antibodies. He had previously been treated with cyclophosphamide, and his cumulative dose of cyclophosphamide was approximately $20 \mathrm{~g}$. He was initially treated with corticosteroids at a dose of $1 \mathrm{mg} / \mathrm{kg}$ and a cycle of rituximab (MabThera, Hoffmann-La Roche, Ltd.) given as 2 intravenous doses of $1 \mathrm{~g}$ two weeks apart. After 3 weeks, his necrotising scleritis was still active, despite his B cell count having fallen to 0 cells $/ \mathrm{mm}^{3}$. We were reluctant to continue his high-dose corticosteroids as he had osteoporosis confirmed on DEXA scanning, and treatment with cyclophosphamide was not felt desirable in view of his cumulative dose. Therefore, infusions of infliximab (Remicade, Merck \& Co.) were arranged and given as doses of $5 \mathrm{mg} / \mathrm{kg}$ at baseline, 2, 6 and 12 weeks. His necrotising scleritis was controlled by week 6 ( fig. 2), and his systemic corticosteroids were reduced to $5 \mathrm{mg}$ prednisolone/day by week 12 . He had his final infusion of infliximab at this point and thereafter was maintained on $5 \mathrm{mg}$ prednisolone/day alone for the following 12 months.

\section{Discussion}

We report a case of necrotising scleritis secondary to GPA, successfully treated with infliximab whilst waiting for rituximab to induce disease remission. Importantly, this enabled cyclophosphamide to be avoided in the acute phase, as rituximab can take up to 6 months to work $[4,5]$. This was especially important as the patient had had significant previous exposure to cyclophosphamide, increasing his risk of malignancy with further treatment. Infliximab rather than etanercept was used as the latter has previously been proven not to be effective for the maintenance of remission in patients with GPA [5, 9]. In addition, retrospective studies of patients with uveitis treated with TNF- $\alpha$ blockade have indicated infliximab to be the more effective treatment in ophthalmic disease [10]. 
There was some concern that the use of two biological agents simultaneously could lead to life-threatening infection or malignancy, but there is little in the literature that addresses this directly. One recent registry-based study of patients being treated with biological agents for rheumatoid arthritis showed their rate of hospitalised infection to be 4.6 per 100 person-years, increasing to 7.0 per 100 person-years for the group that had 'switched' from a different biological agent [11], the situation most analogous to the one here. Interestingly, the rate for rituximab was higher at 10.4 episodes per 100 person-years in this study, falling to 7.1 in switchers; the authors suggested that this might represent higher-risk patients being channelled towards rituximab therapy. Encouragingly for the treating clinician, analysis showed that patient characteristics were a more powerful determinant of the rate of serious infections than specific biological drugs or patterns of switching drugs. Similarly, a meta-analysis of patients treated with TNF- $\alpha$ blockade in rheumatoid arthritis did not demonstrate an increased risk of malignancy [12].

Nevertheless, this case report illustrates the potential usefulness of infliximab in gaining rapid disease control and allowing time for rituximab to take effect, at least in some patients with necrotising scleritis. It also illustrates the increasing treatment options which are available to treat patients with refractory ocular inflammation, and supports the management of such patients in a multidisciplinary setting so that close contact with appropriate specialists can enable these treatments to be taken advantage of by patients in a safe and controlled environment.

\section{Acknowledgements}

The authors gratefully acknowledge the assistance of Ruth Pepper, Alan Salama and Charles Pusey of the Imperial College Healthcare NHS Trust Multidisciplinary Vasculitis Clinic at the Hammersmith Hospital.

\section{Disclosure Statement}

S.R.J.T. was supported by the UK National Institute of Health Research. This work was supported by the Imperial NIHR Comprehensive Biomedical Research Centre. The sponsor or funding organisation had no role in the design or conduct of this research. The authors report no conflicts of interest. This study was approved by the Hammersmith Hospital and Queen Mary's Hospital Research Ethics Committee. 


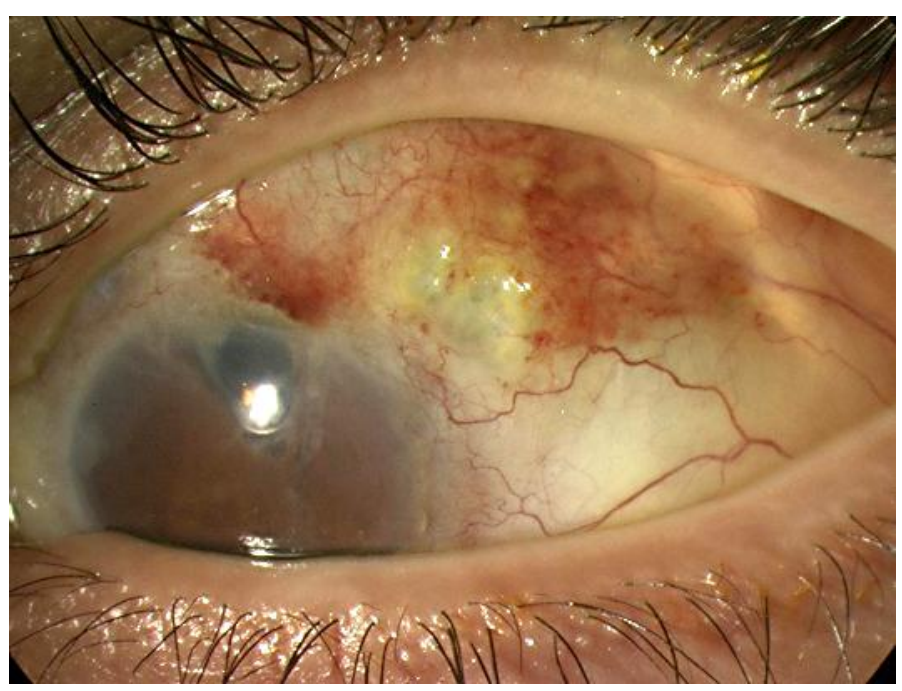

Fig. 1. An active area of necrotising scleritis is visible in the superotemporal quadrant of the left eye, despite $1 \mathrm{mg} / \mathrm{kg} /$ day prednisolone and 2 recent doses of rituximab.

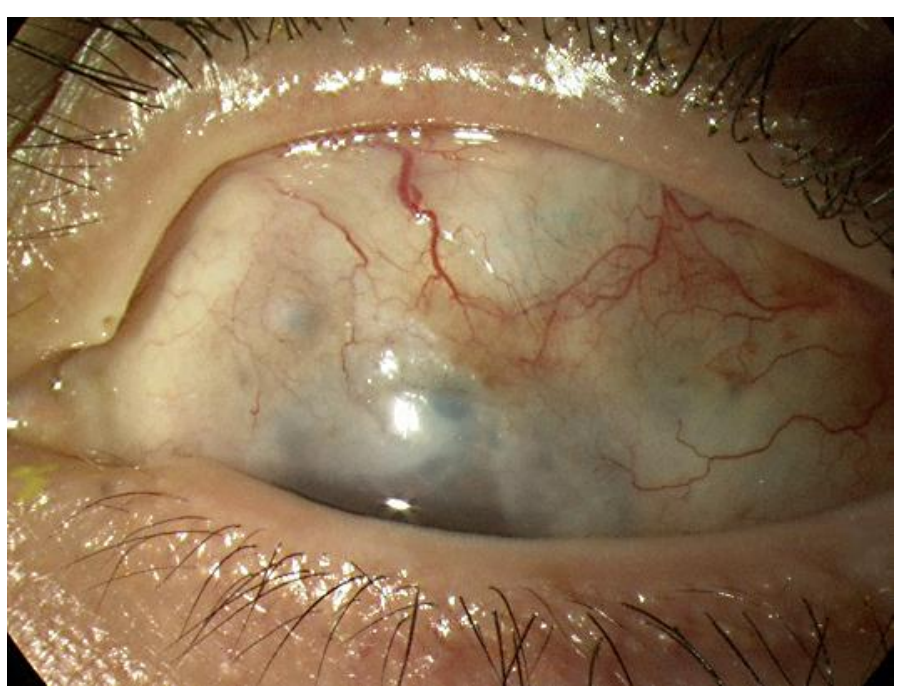

Fig. 2. Six weeks after the institution of infliximab therapy, the necrotic area can be seen to have revascularised and healed. 


\section{References}

1 Taylor SRJ, et al: Ocular manifestations of Wegener's granulomatosis. Expert Rev Ophthalmol 2007;2:91-103.

-2 Sainz de la Maza M, et al: Clinical characteristics of a large cohort of patients with scleritis and episcleritis. Ophthalmology 2012;119:43-50.

3 Stone JH, et al: Rituximab versus cyclophosphamide for ANCA-associated vasculitis. N Engl J Med 2010;363:221-232.

4 Taylor SR, et al: Rituximab is effective in the treatment of refractory ophthalmic Wegener's granulomatosis. Arthritis Rheum 2009;60:1540-1547.

5 Joshi L, et al: Rituximab in refractory ophthalmic Wegener's granulomatosis: PR3 titers may predict relapse, but repeat treatment can be effective. Ophthalmology 2011;118:2498-2503.

-6 Morgan MD, et al: Addition of infliximab to standard therapy for ANCA-associated vasculitis. Nephron Clin Pract 2011;117:c89-c97.

7 Doctor P, et al: Infliximab for the treatment of refractory scleritis. Br J Ophthalmol 2010;94:579-583.

8 Kontkanen M, Paimela L, Kaarniranta K: Regression of necrotizing scleritis in Wegener's granulomatosis after infliximab treatment. Acta Ophthalmol 2010;88:e96-e97.

$\checkmark 9$ Wegener's Granulomatosis Etanercept Trial (WGET) Research Group: Etanercept plus standard therapy for Wegener's granulomatosis. N Engl J Med 2005;352:351-361.

10 Galor A, et al: Differential effectiveness of etanercept and infliximab in the treatment of ocular inflammation. Ophthalmology 2006;113:2317-2323.

11 Curtis JR, et al: The comparative risk of serious infections among rheumatoid arthritis patients starting or switching biological agents. Ann Rheum Dis 2011;70:1401-1406.

12 Leombruno JP, Einarson TR, Keystone EC: The safety of anti-tumour necrosis factor treatments in rheumatoid arthritis: meta and exposure-adjusted pooled analyses of serious adverse events. Ann Rheum Dis 2009;68:1136-1145. 